\title{
Posterior Reversible Encephalopathy Syndrome in a Pediatric Patient with SARS-CoV-2 Infection
}

\author{
Anita Mathew, $D O^{1}$, Richard Sultan, $D O^{2}$ and Zelda J Ghersin, $M D^{3^{*}}$ \\ ${ }^{1}$ Department of Pediatrics, K. Hovnanian Children's Hospital, USA \\ ${ }^{2}$ Department of Pediatric Neurology, K. Hovnanian Children's Hospital, USA \\ ${ }^{3}$ Department of Pediatric Critical Care, K. Hovnanian Children's Hospital, USA
}

\begin{abstract}
Posterior reversible encephalopathy syndrome (PRES) is a clinical syndrome associated with certain underlying conditions or medications, with a spectrum of neurologic findings and characteristic neuroimaging. We describe a case of PRES in a 3-year-old girl positive for SARS-CoV-2 with no other underlying risk factors for the development of this syndrome. We review and suggest plausible mechanisms of neurologic involvement in patients positive for SARS-CoV-2.
\end{abstract}

Keywords

PRES, SARS-CoV-2, Status epilepticus

\section{Introduction}

The severe acute respiratory syndrome coronavirus 2 (SARS-CoV-2) has affected more than 100 million people throughout the world. Thirty-two million cases have been reported in the United States [1] and as of April 2021, more than $22 \%$ of all cases have occurred in children less than 18 [2]. Although most children are asymptomatic, some children present with acute respiratory disease while others present weeks after initial infection with a post-viral illness that affects multiple organ systems, now known as multisystem inflammatory syndrome in children (MIS-C). In patients with SARS-CoV-2, as well as those who meet criteria for MIS-C, neurologic manifestations secondary to exposure are increasingly described. It has been reported that up to $22 \%$ of children admitted with positive SARS-CoV-2 test results have neurologic involvement [3]. Manifestations range from mild symptoms, such as headache, to more severe symptoms including seizures, encephalitis, Guillain-Barre syndrome, encephalopathy, stroke, and acute fulminant cerebral edema [3-5]. It is hypothesized that these neurologic manifestations are either due to direct neuronal viral invasion, or secondary to immune dysregulation and hypercytokinemia with subsequent effects on the central nervous system [6,7]. As the COVID-19 pandemic evolves, it is reasonable to anticipate increased reports of both acute and chronic neurologic sequela.

In the adult population, there have been reports of patients with coronavirus disease 2019 (COVID-19) who developed posterior reversible encephalopathy syndrome (PRES) [8]. It has also recently been reported that a 10 -year-old boy with COVID-19 treated with dexamethasone developed PRES in the setting of hypertension [9]. PRES is a clinical syndrome with a spectrum of heterogenous neurologic manifestations characterized by certain radiographic features. It is secondary to rapid increases in blood pressure, the use of certain medications, renal disease, sepsis, or autoimmune disorders [10]. Neuro-radiographic features suggestive of PRES include symmetric hyperintense lesions in T2-weighted or fluidattenuated inversion recovery (FLAIR) sequences consistent with vasogenic edema, most commonly in the parieto-occipital distribution [10]. Clinically, PRES may present with rapid onset headache, vision changes, seizures and altered mental status, amongst other neurologic findings. PRES is reversible once the underlying etiology is treated, or the offending medication removed. There are several proposed hypotheses regarding the underlying pathophysiology of PRES, but in general, it is thought to be secondary to cerebral vascular auto-dysregulation or endothelial dysfunction $[10,11]$.

*Corresponding author: Zelda J Ghersin, MD, Department of Pediatric Critical Care, K. Hovnanian Children's Hospital, Hackensack Meridian School of Medicine, 1945 State Route 33, Neptune, NJ 07753, USA, Tel: 732-776-4985, Fax: 732-776-2334 Accepted: October 18, 2021

Published online: October 20, 2021

Citation: Mathew A, Sultan R, Ghersin ZJ (2021) Posterior Reversible Encephalopathy Syndrome in a Pediatric Patient with SARS-CoV-2 Infection. J Pediatr Neurol Neurosci 5(2):175-178 
We report a case of a previously well 3-year-old female positive for SARS-CoV-2, who presented in status epilepticus with clinical course and neuroimaging consistent with PRES. To our knowledge, this is the first case of PRES in a pediatric patient with SARS-CoV-2 infection who had no other underlying risk factors for the development of PRES.

\section{Patient Presentation}

A previously healthy, under-immunized, 3-year-old female presented with seizure to a community emergency department. En route in the ambulance she was given 0.5 $\mathrm{mg}$ of Lorazepam. Upon arrival, she was unresponsive with a fixed, upward deviated gaze. An additional $1 \mathrm{mg}$ of Lorazepam was administered, after which the seizure aborted. Per parent, within a three-hour period, the patient suffered four tonic-clonic seizures, each lasting approximately five minutes without return to baseline in between.

The patient had been in her usual state of health until the day prior to admission when she developed a headache. Per report, she had a normal appetite without cough, congestion, fever, rash, vomiting or diarrhea. Notably, one month prior to admission, the patient tested positive for SARS-CoV-2 but was asymptomatic. Her medical history was significant for full term birth via an emergent c-section with a course complicated by meconium aspiration and an episode of seizure in the immediate post-natal period. She remained in the neonatal intensive care unit for two weeks and was subsequently discharged without any anti-epileptic medications or recommended neurologic follow up. Since then, she had been seizure-free and healthy, with normal growth and development.

At the time of presentation to the ED, the patient had a temperature of $96 \mathrm{~F}$, heart rate of 140 and a blood pressure of $100 / 62\left(83^{\text {rd }} / 89^{\text {th }}\right.$ percentile). She was without respiratory distress and had normal oxygen saturation on room air. Due to concern for meningoencephalitis, she was started on Ceftriaxone and Vancomycin at meningitic dosing. Initial laboratory results were significant for a white blood cell count of 10,600 cells per micrometer, with $19 \%$ segmented neutrophils, $2 \%$ band cells and $70 \%$ lymphocytes. A basic metabolic panel was grossly unremarkable (sodium 137 $\mathrm{mmol} / \mathrm{L}$, potassium $4.5 \mathrm{mmol} / \mathrm{L}$, chloride $104 \mathrm{mmol} / \mathrm{L}$, BUN $6 \mathrm{mg} / \mathrm{dL}$, creatinine $0.37 \mathrm{mg} / \mathrm{dL}$, anion gap $8 \mathrm{mmol} / \mathrm{L}$ ). Liver function panel was within normal limits. A creatinine kinase was elevated at $259 \mathrm{iU} / \mathrm{L}$. The patient was positive for SARSCoV-2 via nasopharyngeal nucleic acid amplification testing (NAAT). Non-contrast head CT was performed which showed no acute intracranial pathology. Urine toxicology, EKG and chest X-ray were unremarkable.

The patient was subsequently transferred to our center. Upon arrival to the pediatric intensive care unit, she was hemodynamically stable and breathing without support on room air. The patient was sleepy, but arousable. Her neurological exam was grossly unremarkable with normal distal reflexes bilaterally. On exam, she was noted to have a herpetic lesion on her lower lip and Acyclovir was started. A lumbar puncture was performed, revealing a glucose of 59 $\mathrm{mg} / \mathrm{dL}$, a protein of $24 \mathrm{mg} / \mathrm{dL}$, and a white blood cell count of $1 / \mu \mathrm{L}$. Gram stain was negative, and cultures were no growth after 5 days. CSF studies were also negative for HSV-1 and 2 , an autoimmune encephalitis panel and a viral encephalitis panel. Despite having a positive SARS-CoV-2 from the outside hospital, an expanded respiratory viral panel including the novel SARS-CoV-2 via polymerase chain reaction (PCR) was performed but returned negative. Serology was not sent. The patient did not meet diagnostic criteria for MIS-C (as she was afebrile and without multiple organ system involvement), thus no further workup was initiated. The patient was started

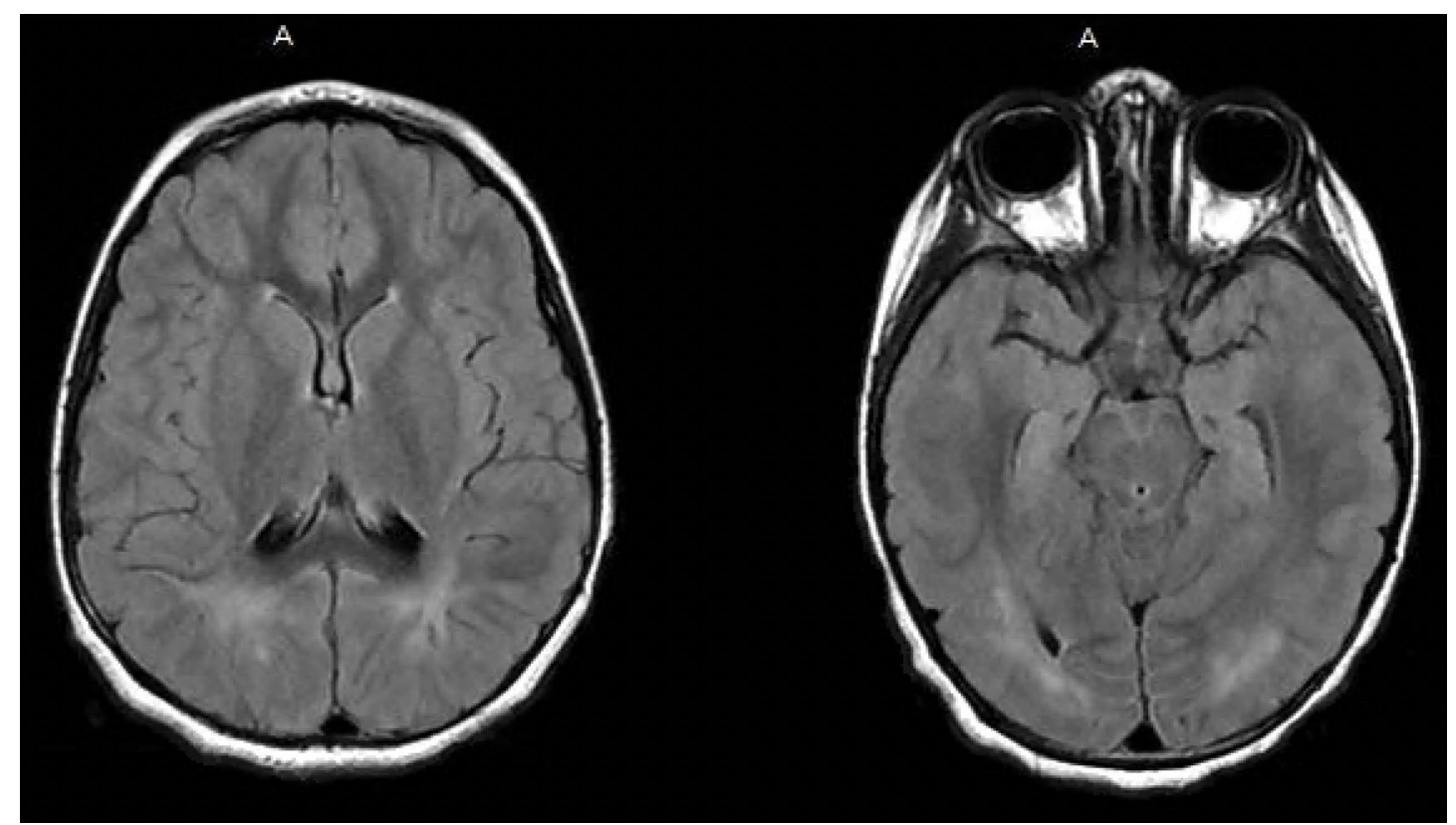

Figure 1: Axial T2 flair imaging demonstrating symmetric increased FLAIR signal in the subcortical white matter of the parieto-occipital lobes. 
on Levetiracetam and placed on continuous video EEG, which did not show further seizure activity. On hospital day two, magnetic resonance imaging of the brain was obtained and was significant for symmetric increased FLAIR signal in the subcortical white matter of the parieto-occipital lobes bilaterally with no associated enhancement or diffusion restriction consistent with PRES (Figure 1). Since discharge the patient has been weaned off Levetiracetam and remains seizure-free.

\section{Discussion}

To our knowledge, this is the first pediatric case of PRES in a patient with SARS-CoV-2 infection without other risk factors for the development of this syndrome. Our patient had no history of hypertension and was normotensive throughout her stay. She had no evidence of renal disease, was not on any medications, and had no known underlying autoimmune disorder that could predispose her to the development of PRES. Without other plausible alternatives, we hypothesize that her clinical presentation and radiographic findings suggestive of PRES are associated with SARS-CoV-2 infection. The patient tested positive for SARS-CoV-2 one month prior to admission (per parental report) and on admission to the outside hospital emergency department by nasopharyngeal NAAT. Interestingly, on admission to the pediatric intensive care unit, an expanded respiratory panel via PCR which includes SARS-CoV-2 was negative possibly due to poor sample collection.

The underlying mechanism(s) of neurologic involvement in SARS-CoV-2 infection are not well understood. It has been suggested that either viral proteins or direct viral invasion into neuronal tissue may predispose patients to develop neurologic and psychiatric complications in the setting of SARS-CoV-2 exposure. Although this has yet to be established in humans, in the mouse model, the SARS-CoV-2 spike protein was detected within the olfactory nerve and viral mRNA has been found in the cerebral spinal fluid of mice, suggesting the virus is able to cross the blood brain barrier [12]. Although the ACE2 receptor is minimally expressed in the brain, SARSCoV-2 achieves host cell entry through its ability to bind angiotensin-converting enzyme 2 (ACE2) receptors $[13,14]$, which has been found on supporting cells such "as pericytes which are involved in blood pressure regulation, blood-brain barrier maintenance and inflammatory responses" $[15,16]$. It is therefore plausible that viral entry via supporting neuronal cells, such as pericytes, may have led to cerebral vascular auto dysregulation and/or endothelial dysfunction predisposing our patient to develop PRES.

Though there have been several cases of PRES in adult patients with COVID-19, it is often associated with labile blood pressures [17] , in line with the typical causative factors of PRES. Montiel, et al. demonstrated that SARS-CoV-2 can infect human endothelial cells [18]. This endothelial dysfunction may lead to elevated pressures and weakening of the blood-brain barrier, with ultimate destruction to the brain's natural ability to auto-regulate its own circulation [19]. However, since our patient did not have significant, sustained variations in blood pressure, this may no longer be sufficient to explain the emergence of PRES in patients with SARSCoV-2. Furthermore, these adult patients had considerable comorbidities, including existing hypertension, obesity, and malignancy [17]; our patient had none. Instead, it appears that by the various mechanisms described above, SARS-CoV-2 infection may have resulted in PRES and subsequent status epilepticus.

Future research is needed to investigate the causal relationship between SARS-CoV-2 infection in cells and the subsequent changes that could result in the clinical course and associated neuro-radiologic findings of our patient. It appears clear that SARS-CoV-2 has a unique mechanism of affecting the central nervous system, however, our case illustrates the importance of exploring the underlying pathophysiology to better understand the varied neurologic manifestations associated with infection. Though our patient seems to have no permanent neurological deficits, which is consistent with PRES, it is critical that clinicians remain on high alert for neurologic complications of SARS-CoV-2 infection.

\section{Conflicts of Interests Statement}

Authors have no conflicts to report.

\section{Financial Support}

None.

\section{References}

1. Johns Hopkins Coronavirus Resource Center (2021) COVID-19 Dashboard by the Center for Systems Science and Engineering (CSSE) at Johns Hopkins University (JHU).

2. https://covid.cdc.gov/covid-data-tracker/\#demographics

3. LaRovere KL, Riggs BJ, Poussaint TY, et al. (2020) Neurologic involvement in children and adolescents hospitalized in the United States for COVID-19 or Multisystem Inflammatory Syndrome. JAMA Neurol 78: 536-547.

4. Feldstein LR, Rose EB, Horwitz SM, et al. (2020) Multisystem inflammatory syndrome in U.S. children and adolescents. N Engl J Med 383: 334-346.

5. Lin JE, Asfour A, Sewell TB, et al. (2021) Neurological issues in children with COVID-19. Neurosci Lett 743: 135567.

6. Coperchini F, Chiovato L, Croce L, et al. (2020) The cytokine storm in COVID-19: An overview of the involvement of the chemokine/ chemokine-receptor system. Cytokine Growth Factor Rev 53: 25 32.

7. Mehta P, McAuley DF, Brown M, et al. (2020) COVID-19: Consider cytokine storm syndromes and immunosuppression. Lancet 395: 1033-1034.

8. Anand P, Lau KHV, Chung DY, et al. (2019) Posterior reversible encephalopathy syndrome in patients with coronavirus disease 2019: Two cases and a review of the literature. J Stroke Cerebrovasc Dis 29: 105212.

9. Korkmazer B, Ozugul M, Hikmat E, et al. (2021) Posterior reversible encephalopathy syndrome in a pediatric COVID-19 patient. Pediatr Infect Dis J 40: e240-e242.

10. Fischer M, Schmutzhard E (2017) Posterior reversible encephalopathy syndrome. J Neurol 264: 1608-1616. 
11. Hinduja A (2020) Posterior reversible encephalopathy syndrome: clinical features and outcome. Front Neurol 11: 71.

12. Rhea EM, Logsdon AF, Hansen KM, et al. (2021) The S1 protein of SARS-CoV-2 crosses the blood-brain barrier in mice. Nat Neurosci 24: 368-378.

13. Guo J, Huang Z, Lin L, et al. (2020) Coronavirus disease 2019 (COVID-19) and cardiovascular disease: A viewpoint on the potential influence of angiotensin-converting enzyme inhibitors/ angiotensin receptor blockers on onset and severity of severe acute respiratory syndrome coronavirus 2 infection. J Am Heart Assoc 9: e016219.

14. Labò N, Ohnuki H, Tosato G (2020) Vasculopathy and coagulopathy associated with SARS-CoV-2 infection. Cells 9: 1583.
15. Brann DH, Tsukahara T, Weinreb C, et al. (2020) Non-neuronal expression of SARS-CoV-2 entry genes in the olfactory system suggests mechanisms underlying COVID-19-associated anosmia. Sci Adv 6: e5801.

16. Brown LS, Foster CG, Courtney JM, et al. (2019) Pericytes and neuromuscular function in the healthy and diseased brain. Front Cell Neurosci 13: 282.

17. Franceschi AM, Arora R, Wilson R, et al. (2020) Neurovascular complications in COVID-19 infection: Case series. AJNR Am J Neuroradiol 41: 1632-1640.

18. Monteil V, Kwon H, Prado P, et al. (2020) Inhibition of SARSCoV-2 infections in engineered human tissues using clinicalgrade soluble human ACE2. Cell 181: 905-913.

19. Kishfy L, Casasola M, Banankhah P, et al. (2020) Posterior reversible encephalopathy syndrome (PRES) as a neurological association in severe Covid-19. J Neurol Sci 414: 116943. 